This article was published in Biomedicine and Pharmacotherapy, 81, 273-280, 2016 http://dx.doi.org/10.1016/j.biopha.2016.04.030

\title{
Long-term exercise training as a modulator of mammary cancer vascularization
}

A.I. Faustino-Rocha ${ }^{\mathrm{a}, b, c, d, *}$, A. Silva ${ }^{\mathrm{e}}$, J. Gabriel $^{\mathrm{e}}$, R.M. Gil da Costa ${ }^{\mathrm{f}, \mathrm{g}}, \mathrm{M}$. Moutinho ${ }^{\mathrm{a}}$, P.A. Oliveira ${ }^{a, b}$, A. Gama ${ }^{a, c}$, R. Ferreira ${ }^{d}$, M. Ginja ${ }^{a, b}$

a Department of Veterinary Sciences, School of Agrarian and Veterinary Sciences, University of Trás-os-Montes and Alto Douro (UTAD), Vila Real, Portugal

$\mathrm{b}$ Center for the Research and Technology of Agro-Environmental and Biological Sciences (CITAB), UTAD, Vila Real, Portugal

c Animal and Veterinary Research Center (CECAV), UTAD, Vila Real, Portugal

d Organic Chemistry, Natural Products and Foodstuffs (QOPNA), Mass Spectrometry Center, Department of Chemistry, University of Aveiro, Aveiro, Portugal

e Institute of Science and Innovation in Mechanical and Industrial Engineering (INEGI), Faculty of Engineering (FEUP), University of Porto, Porto, Portugal

f Laboratory for Process Environment Biotechnology and Energy Engineering (LEPABE), FEUP, University of Porto, Porto, Portugal

g Molecular Oncology and Viral Pathology Group, CI-IPOP, Portuguese Institute of Oncology, Porto, Portugal

\footnotetext{
Abstract

Background: Breast cancer remains a leading cause of death by cancer worldwide. It is commonly accepted that angiogenesis and the expression of angiogenic factors such as vascular endothelial growth factor-A (VEGF-A) is associated with the increased risk of metastasis and poor patient outcome.

Objective: This work aimed to evaluate the effects of long-term exercise training on the growth and vascularization of mammary tumors in a rat model.

Materials and methods: Fifty female Sprague-Dawley rats were divided into four groups: two $N$-methyl- $N$ - nitrosourea (MNU)-exposed groups (exercised and sedentary) and two control groups (exercised and sedentary). MNU was administered once, intraperitoneally at 7 weeks-old. Animals were then exercised on a treadmill for 35 weeks. Mammary tumors were evaluated using thermography, ultrasonography [Power Doppler (PDI), B Flow and contrast-enhanced ultrasound (CEUS)], and immunohistochemistry (VEGF-A).

Results: Both, MNU sedentary and exercised groups showed $100 \%$ of tumor incidence, but exercised animals showed less tumors with an increased latency period. Exercise training also enhanced VEGF-A immunoexpression and vascularization (microvessel density, MVD) $(p<0.05)$, and reduced histological aggressiveness. Ultrasound and
} 
thermal imaging analysis confirmed the enhanced vascularization of tumors on exercised animals.

Conclusion: Long-term exercise training increased VEGF-A expression, leading to enhanced tumor vascularization and reduced tumor burden, multiplicity and histological aggressiveness.

\section{Introduction}

Despite recent advances in diagnostic and therapeutic approaches, breast cancer remains one of the leading causes of death by cancer worldwide [1,2]. Angiogenesis, the formation of new blood vessels from pre-existing vessels and vascular endothelial cells, is essential for tumor growth, by supplying nutrients and oxygen [3,4]. Angiogenesis is regulated by a balance between proangiogenic and antiangiogenic factors, produced by both tumor and host cells, namely endothelial cells, pericytes and leokocytes [5]. Vascular endothelial growth factor (VEGF) is the most potent and widely distributed angiogenic factor [6]. The VEGF family is composed of several members - VEGF-A， B, C, D and E - of which VEGF-A is the most potent [7]. VEGF-A stimulates endothelial cell proliferation and migration, prevents the regression of newly formed vessels and increases microvascular permeability [5;6]. VEGF-A expression has been associated with cancer progression, increased risk of metastasis and poor outcome of lung, esophagus, colorectal and breast cancer $[8,9]$. Tumor vascularization can be non-invasively assessed by imaging tools such as ultrasonography and thermography [10]. Ultrasonography is very useful in women with dense breasts and in the characterization of breast lesions identified in mammographic examination [11], being frequently used as an adjuvant tool for clinical breast examination. Thermography measures the infrared radiation emitted from the body, revealing superficial temperature patterns which are directly related to local vascularization, and may therefore be used to study physiological and pathological vascular changes [12-14]. This technique was first introduced for breast cancer screening in 1956 and later recognized by the Food and Drug Administration as a tool for breast cancer risk assessment [15].

It is largely accepted that exercise training exerts a beneficial effect in some lymphomas and in colon, lung, endometrial, prostate and breast cancer [16-23]. Systematic reviews have concluded that the practice of physical activity in cancer patients improves important clinical (quality of life and fatigue) and physiological outcomes (muscle strength) [24]. Furthermore, several investigators have studied the effects of exercise training on the biopathology of mammary tumors themselves. However, these studies have focused their attention on the effects of shorter exercise training protocols using xenograft models [25-27]. Despite their usefulness and widespread application, xenograft models show important limitations, related to the lack of a functional immune system and of the complex tumor cell population which, in spontaneous tumors, evolves through a lengthy multi-step process of carcinogenesis. In particular, xenograft models are considered too artificial for studying tumor angiogenesis, and more realistic models are being called for [28]. This work intends to address these concerns, by choosing a mammary cancer model induced in immune-competent rats by $N$ - methyl- $N$-nitrosourea (MNU). We hypothesized that exercise training can modulate the microenvironment of mammary tumors, and thus it was studied the effects of long-term exercise training on 
tumor growth and vascularization, employing thermography, ultrasonography and immunohistochemical techniques.

\section{Materials and methods}

\subsection{Animals}

Fifty female Sprague-Dawley rats, with 4-5 weeks of age were obtained from Harlan Interfauna Inc. (Barcelona, Spain). Animals were housed at the facilities of the University of Trás-os-Montes and Alto Douro in filter-capped polycarbonate cages with corncob for bedding under controlled conditions of temperature $\left(23 \pm 2{ }^{\circ} \mathrm{C}\right)$, humidity $(50 \pm 10 \%)$, air system filtration (10-20 ventilations/ hour) and on a $12 \mathrm{~h}: 12 \mathrm{~h}$ light:dark cycle. Tap water and a basic standard laboratory diet (4RF211, Mucedola, Italy) were supplied ad libitum during the study. Cages were cleaned and water was changed once per week. All procedures were done in accordance with European and National Legislation (European Directive 2010/ 63/EU and National Decree-law 113/2013). The Portuguese Ethics Committee for Animal Experimentation approved all the experiments and procedures carried out on the animals (Direcção-Geral de Alimentação e Veterinária, Approval no. 008961).

\subsection{Animal experiments}

After one week of quarantine, animals were allowed to acclimate to laboratory conditions for two weeks. Then, they were randomly divided into four experimental groups: MNU sedentary $(n=15)$, MNU exercised $(n=15)$, control sedentary $(n=10)$ and control exercised $(n=10)$. The development of mammary tumors was induced in animals from both MNU sedentary and MNU exercised groups by a single intraperitoneal administration of the carcinogen agent MNU (Isopac, lot 100M1436 V, Sigma Chemical Co., Madrid, Spain) at a dose of $50 \mathrm{mg} / \mathrm{kg}$, at seven weeks of age. MNU was used within one hour after its preparation. Animals from control groups received a single administration of the vehicle (saline solution $0.9 \%$ ). After this, animals from exercised groups were acclimated to the treadmill running (Treadmill Control $\mathbb{R}$ LE 8710, Panlab, Harvard Apparatus, USA) for a five-day period at a speed of $20 \mathrm{~m} / \mathrm{min}$ increasing progressively from 20 to $60 \mathrm{~min} /$ day. Then, the duration of the exercise was maintained as $60 \mathrm{~min} /$ day, 5 times/week during 35 weeks. The animals were daily observed to monitor their general health status. They were weekly palpated for the detection of mammary tumors development. The animals' body weight was measured weekly using a top-loading scale (Mettler PM 4000, LabWrench, Midland, Canada). The day of the MNU administration was considered the first day of the study and the animals' sacrifice 35 weeks later was considered the end of the study. At the end of the study, the accurate body weight was calculated by subtracting the tumors' weight to the total body weight, and the mortality index (MI) was calculated using the following equation:

$$
M I(\%)=\frac{\text { number of animals that died during the study }}{\text { number of animals at the beginning of the study }} \times 100
$$


Twenty-four hours before the examination, the skin overlying the mammary tumors was shaved using a machine clipper (AESCULAP R GT420 Isis, Aesculap Inc, Center Valley, PA, USA). At the end of the experimental protocol, immediately before the animals' sacrifice, the mammary tumors were evaluated by thermography and ultrasonography. For these examinations, all survived animals were anesthetized by intraperitoneal administration of ketamine (75 mg/kg; Imalgene 1000, lot LBF133BB, Merial S.A.S., Lyon, France) and xylazine (10 mg/kg; Rompun 2\%, lot KPO78 x 0, Bayer Healthcare S.A., Kiel, Germany).

\subsection{Thermographic evaluation}

The thermographic evaluation was performed using a far infrared camera from FLIR $\mathbb{R}$ model A325 (USA), with a sensitivity of $68 \mathrm{mK}$ and a spatial resolution of 320x240 pixels. The images were recorded at one frame per second for future analyses but the integration time for the micro bolometer was approximately $16.6 \mathrm{~ms}$. Animals were manually held and filmed at a constant distance $(0.35 \mathrm{~m})$. The animal emissivity was set to 0.98 and the tumor borders were marked to overlap with a visible image [29]. Representative frames were selected and analyzed using the ThermaCam Researcher Pro 2.10 (FLIR Systems, Inc., USA) software. In this analysis, the maximum, minimum and average temperatures of each region of interest were obtained. These measurements reflect the vascularization and the extension of necrotic areas of mammary tumors. Higher vascularized tumors are expected to exhibit less extensive necrotic areas and consequently to present higher maximum, minimum and mean temperature, and lower thermal amplitude. The opposite is expected to poor vascularized tumors.

\subsection{Ultrasonographic evaluation}

For ultrasonographic analysis it was used a real-time scanner (Logic P6 ${ }^{\mathbb{R}}$, General Electric Healthcare, Milwaukee, WI, USA) and a $10 \mathrm{MHz}$ linear transducer with a standoff pad (Sonokit1, MIUS Ltd, Gloucestershire, England). The animals were placed in supine position and it was applied acoustic gel (Aquasonic $\mathbb{R}$, Parker Laboratories Inc, Fairfield, New Jersey, USA), the ultrasonographic images using Power Doppler (PDI) and B Flow modes were obtained in sagittal planes using light pressure to avoid the distortion of tumors' shape. The images were recorded and the color pixels density (CPD) in PDI and B Flow images was determined according to the formula previously published by Denis et al. [30], using Adobe Photoshop version 7.0.

\subsection{Necropsy}

Following ultrasonographic examination, all survived animals were humanely sacrificed by exsanguination by cardiac puncture as indicated by Federation for Laboratory Animal Science Associations [31]. All animals were scalped and the skin was carefully observed under a light for the detection of small mammary tumors. All tumors were removed and weighed. Mammary tumors' volume was calculated based on tumors' weight applying a 
previously published formula [32]. All mammary tumors and organs were fixated in buffered formalin during $24 \mathrm{~h}$.

\subsection{Histologyand immunohistochemistry}

After fixation, mammary tumors were routinely processed for histological analysis; 2 $\mu \mathrm{m}$-thick sections were stained with hematoxylin and eosin (H\&E) and histologically classified by a pathologist according to the classification previously established by Russo and Russo [33]. Each mammary tumor was classified according to the histological pattern with higher proportion in each tumor section. The mean area of necrosis was quantified in each histological section. For this purpose, three different fields were randomly selected and analyzed using ImageJ software (US National Institutes of Health, Maryland, USA).

The immunohistochemical detection of VEGF-A was performed using the standard NovoLink Polymer Detection System protocol (Leica Biosystems, Newcastle, UK).

Sections were incubated overnight at $4{ }^{\circ} \mathrm{C}$ with a primary antibody for VEGF-A (clone JH121, Merck Millipore, Darmstadt, Germany) at a dilution of 1:100. The VEGF-A immunoexpression was semi-quantitatively and quantitatively assessed. For the semiquantitative way, a minimum of 1000 neoplastic cells were evaluated in each mammary tumor and the VEGF-A immunoexpression was assessed according to five levels: grade 0 (no staining detected in tumor cells), grade 1 (1-24\% of tumor area showed positive staining), grade 2 (25-49\% of tumor stained), grade 3 (50-75\% of tumor stained) and grade $4>75 \%$ of tumor stained). The staining intensity was also evaluated as: level 0 (unstained), + (weak staining), ++ (moderate staining) and +++ (intense staining) [34]. For the quantitative way, a representative image from each tumor was taken with a 40x objective and the VEGF immunoexpression was quantified using an Image Manipulation Program 2.8 (GIMP 2.8, CNE, Free Software Foundation, Boston, USA) [35]. The micro- vessels were counted in the three most vascularized hot spots, in 200x

magnification fields (corresponding to approximately $0.76 \mathrm{~mm}^{2}$ ) from which the mean was obtained in order to determine the microvessel density (MVD). Areas of fibrosis, necrosis and inflammation and vessels with muscular walls were not counted.

\subsection{Statistical analysis}

Continuous data were statistically analyzed with SPSS $\mathbb{R}$ version 17 (Chiçago, IL, USA) using independent sample $t$-test and ANOVA with the Bonferroni correction for multiple comparisons. Histological and immunohistochemical results were analyzed using $\chi^{2}$ tests. Pearson correlation was used to assess the correlation between tumors' volume, temperature (maximum, minimum, mean and thermal amplitude), CPD (for PDI and B Flow) and MVD. All data were expressed as mean \pm standard error (S.E.); $p$-values lower than 0.05 were considered statistically significant. 


\section{Results}

\subsection{General findings}

One animal from MNU exercised group did not adapt to the exercise training and was excluded from the study. During the experiment nine animals died: four animals from the MNU sedentary group ( $\mathrm{MI}=27 \%$ ), four animals from the MNU exercised group (MI $=29 \%)$ and one animal from the control sedentary group $(\mathrm{MI}=10 \%)$. Data from these animals were not included in the study. The final accurate body weight was not statistically different among groups; however, it was slightly lower in MNU groups than in control ones $(p>0.05)$ (data not shown).

\subsection{Mammary tumors}

Animals from control groups did not develop any mammary tumor. All animals from both MNU groups developed mammary tumors (incidence of 100\%). The first mammary tumor was palpated ten weeks after MNU administration in the MNU sedentary group, while the animals from MNU exercised group developed the first mammary tumor two weeks later, at 12th week after MNU administration. At the end of the experiment, the MNU sedentary group developed a total of 28 mammary tumors $(2.55 \pm 1.44$ tumors per animal), while the MNU exercised group developed 23 mammary tumors $(2.30 \pm 1.42$ tumors per animal); the difference did not reach the level of statistical significance ( $p$ $=0.484)$ (Table 1). Although the differences were not statistical significant, the tumors' weight and volume were higher in MNU exercised animals compared with MNU sedentary ones $(p>0.05)$. Similarly, the mean area of necrosis was higher in tumors from exercised animals compared with tumors from sedentary ones $(p>0.05)$ (Table 1). All mammary tumors from both groups were histologically evaluated according to the predominant histological pattern (Table 1). Different histological patterns of nonneoplastic, benign and malignant mammary lesions were identified. Animals from the MNU exercised group developed a higher number of benign lesions $(p=0.034)$ and less malignant lesions $(p=0.123)$ compared with animals from the MNU sedentary group. In both groups, MNU sedentary and MNU exercised, the number of malignant lesions was higher than the number of benign ones, being the papillary noninvasive carcinoma the most frequent histological pattern. It was also important to note that animals from the MNU exercised group did not develop any invasive comedocarcinoma, which was the most aggressive lesion identified in this experimental protocol, while animals from MNU sedentary group developed two invasive comedocarcinomas.

\subsection{Thermographic and ultrasonographic analysis}

Eleven tumors from each MNU group were evaluated by thermography $(\mathrm{n}=11+11)$ and seventeen were evaluated by ultrasonography $(n=17+17)$. The maximum temperature was very similar between groups $(p>0.05)$. Although the differences did not reach the level of statistical significance, the minimum and mean temperatures were slightly higher in MNU sedentary group compared with MNU exercised one; inversely, the thermal amplitude was higher in MNU exercised group $(p>0.05)$. Although the maximum temperature was very 
similar between groups, the lower minimum temperature in MNU exercised group when compared with MNU sedentary one led to a lower mean temperature and higher thermal amplitude in exercised group. This lower minimum temperature in MNU exercised group was probably due to the higher volume of these mammary tumors and the consequent occurrence of more extensive necrotic areas that are characterized by low temperature. The minimum temperature was statistically different from the maximum temperature in both groups MNU sedentary and MNU exercised $(p<0.05)$ (Table 2, Fig. 1), suggesting that the tumors from both experimental groups were not uniform and there were very distinct areas in each one. Some of these areas were highly vascularized with high temperature, and other ones were necrotic with low temperature.

In both groups the CPD detected by B Flow was higher than CPD detected by PDI, however the difference was only statistically significant in MNU exercised group $(p<0.05)$. The CPD detected in MNU exercised group was higher than that detected in MNU sedentary group ( $p$ $>0.05)$. Similarly, the MVD determined in MNU exercised animals was higher than that detected in MNU sedentary animals $(p<0.05)$ (Table 2, Fig. 2). These data together suggest that B Flow mode is more sensitive than PDI in the detection of small blood vessels and that exercise training increased the mammary tumors vascularization (tumors from MNU exercised group were more vascularized that those from animals from MNU sedentary group).

\subsection{Immunohistochemical analysis}

VEGF-A showed a cytoplasmic and homogenous immunolabelling in neoplastic cells (Fig. 3), while endothelial cells exhibited variably intense cytoplasmic VEGF-A immunoexpression. All mammary lesions from both groups exhibited immunolabelling for VEGF-A (no lesions were classified with score 0) (Table 3).

The majority of mammary lesions from MNU sedentary group were classified with score 3 (46.4\% of the lesions) ( $p<0.05$ from scores 1 and 2 ). The score 4 was the most frequently identified in MNU exercised group (47.8\% of the lesions were classified with this score) $(p<0.05$ from score 1) (Table 3$)$. In the same way, the VEGF- A immunoexpression evaluated by GIMP revealed a higher immunoexpression of this marker in tumors from MNU exercised groups compared with tumors from sedentary one $(p>0.05)$ (Table 2).

The staining intensity in the lesions from MNU sedentary group was mainly classified as moderate $(++)(71.4 \%$ of the lesions). This score was statistically different from the remaining scores $(p<0.05$ from weak $(+)$ and intense $(+++))$. Additionally, the number of mammary tumors classified with moderate $(++)$ intensity in MNU sedentary group was higher when compared with those classified with this score in MNU exercised group ( $p$ $<0.05)$. In the MNU exercised group, the staining intensity was mainly classified as weak $(+)$ (this score was attributed to $47.8 \%$ of the lesions) ( $p>0.05$, not statistically different from the remaining scores of intensity) (Table 3 ).

\subsection{Correlations between data}

A significant negative correlation was observed between tumors' volume and minimum temperature, and between thermal amplitude and minimum and mean temperature ( $p<$ 
0.05). A positive significant correlation was observed between tumors' volume and thermal amplitude, and between maximum, mean, and minimum temperature $(p<$ 0.05) (Table 4). Correlation between MVD and tumor vascularization as detected with ultrasound and thermography images, was weak and statistically non-significant $(p>0.05)$.

\section{Discussion}

Breast cancer is the most frequently diagnosed cancer in women worldwide [1]. Angiogenesis is essential for breast cancer growth and invasion [3] but hypoxic tumors often show poor prognosis, developing an aggressive phenotype and resistance to systemic (chemotherapy) and regional (radiotherapy) therapy [24,36]. This resistance occurs due to the lack of oxygen, which is necessary to fixate DNA damage caused by chemotherapy or radiotherapy, and also due to the non-proliferative status of many neoplastic cells in hypoxic microenvironments [37]. Tumor vascularization thus appears as a double-edged sword that requires a deeper understanding before rational modulation approaches may be applied.

In the present study, mortality was associated with the development of mammary tumors in the MNU-exposed groups, and was lower than previously reported in a similar work [38]. MNU-exposed animals also showed a lower accurate body weight compared with control animals, revealing a loss of body condition, which had not been reported in a comparable study [39]. Exercise training increased the latency period and reduced the number of tumors, which may be due to the up-regulation of immunity induced by exercise training [40]. These effects are in line with previous findings from related models $[39,41]$. However, exercise training increased the tumors' weight and volume, which may be due to the enhancement of blood perfusion, as previously reported [42]. A similar observation was made in prostate tumors in exercised mice [36]. Histologically, exercised animals developed more benign and less malignant lesions compared with sedentary animals. However, in both groups, the number of malignant lesions was higher than the number of benign ones. Papillary carcinoma was the most frequently identified histological pattern, in accordance with previous findings $[38,43]$. It worth to note that exercised animals did not develop any invasive comedocarcinoma, the most aggressive lesions diagnosed in sedentary animals, further suggesting that exercise training played a protective effect. Mammography and ultrasonography are well established techniques for breast cancer screening [44], especially when used in combination [44]. It is worth noting that B Flow consistently detected higher CPD than PDI and seems to be a more sensitive technique for assessing tumor vascularization, as previously observed [32]. Concerning thermography, the Society of Breast Imaging reports that no studies show clear benefits when using this technique alone or as an adjunct to mammography [45].

In fact, breast infrared thermography has rarely been used to monitor tumor growth in experimental animals $[46,47]$. The use of thermography for studying cancer is based on the detection of heat generated by the metabolic activity of the proliferating tumor cells and of heat generated by new blood vessels supporting the growth of tumor $[44,48]$. Thus, the process of neoangiogenesis associated with carcinogenesis induces an increase of the skin temperature above the developing tumor [49-51]. In this study, larger tumors were correlated with lower minimum temperature $(p<0.05)$ and increased thermal amplitude $(p<0.05)$, most likely because they had wider central necrotic areas. Previous studies 
also reported a thermographic association between tumor necrosis and reduced surface skin temperature [52-54].

Elevated VEGF-A levels have been correlated with higher proliferation rate, infiltrative growth and poor prognosis in many solid tumors including those of the breast [55], lung [56], colon [57], liver [58], and bladder [59]. In the present study, higher VEGF- A expression was found among exercised animals, and this correlated with higher tumor vascularization, as assessed by MVD and CPD. This is in line with previous studies using lung [60] and breast cancer [24] xenografts, but in contrast with other reports [20,61-65]. Importantly, tumors from the exercised group showed higher VEGF-A expression and vascularization were also histologically less aggressive and longer latency periods. This agrees with findings from canine models [66] and supports the hypothesis that enhanced tumor vascularization may have a beneficial effect and help countering tumor progression.

Although, we hypothesized that long-term exercise training could inhibit mammary tumors' growth and aggressiveness by the inhibition of tumors vascularization, surprisingly we verified that the exercise training promoted tumors vascularization (increased VEGF-A immunoexpression, MVD, and CPD detected by PDI and B Flow) and growth (tumors with higher volume) but reduced the number of mammary tumors and their aggressiveness, and increased latency period.

This study is the first to evaluate the effects of long-term exercise training on an immunocompetent model of mammary tumorigenesis, and comparisons with data from xenograft models should be made cautiously. The present results pave the way for further studies on modulation approaches for tumor vascularization using relevant immunocompetent models, particularly those addressing the relationship between exercise training, angiogenesis and tumor progression.

\section{Conflict of interests}

None.

\section{Acknowledgements}

This work was supported by Portuguese Foundation for Science and Technology (the research project PTDC/DES/114122/2009, the project UID/AGR/04033/2013 and postgraduation grant to A. Faustino SFRH/BD/102099/2014), European Regional Development Found (COMPETE, FCOMP-01-0124-FEDER-014707/022692), Project UID/EQU/00511/2013-LEPABE(Laboratory for Process Engineering, Environment, Biotechnology and EnergyEQU/00511) and Project POCI-01-0145-FEDER-006958 by FEDER funds through Operacional Competitiveness and Internacionalization Programme - COMPETE2020.

\section{References}

[1] A. Jemal, R. Siegel, E. Ward, T. Murray, J.Q. Xu, M.J. Thun, Cancer statistics, 2007, Cancer J. Clin. 57 (2007) 43-66.

[2] R. Siegel, D. Naishadham, A. Jemal, Cancer statistics, 2012, Cancer J. Clin. 62 (2012) 10-29. 
[3] C. Culy, Antiangiogenic cancer therapy, Drugs Today 41 (2005) 23-36.

[4] McDonald, Basic Mechanism to Therapeutic Applications. Michigan. (2000).

[5] L.S. Rosen, VEGF-targeted therapy: therapeutic potential and recent advances, Oncologist 10 (2005) 382-391.

[6] G. Gasparini, Prognostic value of vascular endothelial growth factor in breast cancer, Oncologist 5 (2000) 37-44.

[7] R.A.A. Mohammed, A. Green, S. El-Shikh, E.C. Paish, I.O. Ellis, S.G. Martin, Prognostic significance of vascular endothelial cell growth factors A, C and D in breast cancer and their relationship with angio- and lymphangiogenesis, Br. J. Cancer 96 (2007) 1092-1100.

[8] Z.Y. Hu, C. Fan, C. Livasy, X.P. He, D.S. Oh, M.G. Ewend, L.A. Carey, S. Subramanian, R. West, F. Ikpatt, O.I. Olopade, M. van de Rijn, C.M. Perou, A compact VEGF signature associated with distant metastases and poor outcomes, BMC Med. 7 (2009).

[9] C.W. Qiu, D.G. Lin, J.Q. Wang, C.Y. Li, G.Z. Deng, Expression and significance of PTEN and VEGF in canine mammary gland tumours, Vet. Res. Commun. 32 (2008) 463-472.

[10] E.Y. Ko, S.H. Lee, H.H. Kim, S.M. Kim, M.J. Shin, N. Kim, G. Gong, Evaluation of tumor angiogenesis with a second-generation US contrast medium in a rat breast tumor model, Korean J. Radiol. 9 (2008) 243-249.

[11] P. Crystal, S.D. Strano, S. Shcharynski, M.J. Koretz, Using sonography to screen women with mammographically dense breasts, Am. J. Roentgenol. 181 (2003) 177182.

[12] A. Bhowmik, R. Repaka, S.C. Mishra, Thermographic evaluation of early melanoma within the vascularized skin using combined non-Newtonian blood flow and bioheat models, Comput. Biol. Med. 53 (2014) 206-219.

[13] N. Diakides, J. Bronzino, Medical Infrared Imaging, CRC Press, Florida, 2008. ${ }^{[14]}$ M. Just, C. Chalopin, M. Unger, D. Halama, T. Neumuth, A. Dietz, M. Fischer, Monitoring of microvascular free flaps following oropharyngeal reconstruction using infrared thermography: first clinical experiences, Eur. Arch. Oto Rhino Laryngol. (2015) (Epub ahead of print).

[15] G. Moore, Breast cancer: early detection needed, Bus. Health 19 (2001) 39.

[16] A.S. Yamashita, F.S. Lira, J.C. Rosa, E.C. Paulino, P.C. Brum, C.E. Negrao, R.V. dos Santos, M.L. Batista, C.O. do Nascimento, L.M. Oyama, M. Seelaender, Depotspecific modulation of adipokine levels in rat adipose tissue by diet-induced obesity: the effect of aerobic training and energy restriction, Cytokine 52 (2010) 168-174. [17] E.A. Murphy, J.M. Davis, A.S. Brown, M.D. Carmichael, E.P. Mayer, A. Ghaffar, Effects of moderate exercise and oat beta-glucan on lung tumor metastases and macrophage antitumor cytotoxicity, J. Appl. Physiol. 97 (2004) 955-959.

[18] C. Giannini, T. de Giorgis, A. Mohn, F. Chiarelli, Role of physical exercise in children and adolescents with diabetes mellitus, J. Pediatr. Endocrinol. Metab. 20 (2007) 173-184.

${ }^{[19]}$ K.A. Esser, C.E. Harpole, G.S. Prins, A.M. Diamond, Physical activity reduces prostate carcinogenesis in a transgenic model, Prostate 69 (2009) 1372-1377.

[20] M. Al-Jarrah, I. Matalka, H. Al Aseri, A. Mohtaseb, I. Smirnova, L. Novikova, L. Stehno-Bittel, A. AlKhateeb, Exercise training prevents endometrial hyperplasia and 
biomarkers for endometrial cancer in rat model of type 1 diabetes, J. Clin. Med. Res. 2 (2010) 207-214.

[21] R.Y. Wang, Y.R. Yang, S.M. Yu, Protective effects of treadmill training on infarction in rats, Brain Res. 922 (2001) 140-143.

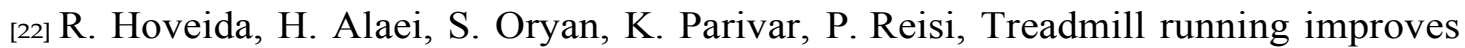
spatial memory in an animal model of Alzheimer's disease, Behav. Brain Res. 216 (2011) 270-274.

[23] S. Marcuzzo, M.F. Dutra, F. Stigger, P.S. do Nascimento, J. Ilha, P.I. Kalil-Gaspar, M. Achaval, Beneficial effects of treadmill training in a cerebral palsy-like rodent model: walking pattern and soleus quantitative histology, Brain Res. 1222 129-140.

[24] L.W. Jones, B.L. Viglianti, J.A. Tashjian, S.M. Kothadia, S.T. Keir, S.J. Freedland, M.

Q. Potter, E.J. Moon, T. Schroeder, J.E. Herndon, M.W. Dewhirst, Effect of aerobic exercise on tumor physiology in an animal model of human breast cancer, J. Appl. Physiol. 108 (2010) 343-348.

[25] C.N. Holick, P.A. Newcomb, A. Trentham-Dietz, L. Titus-Ernstoff, A.J. Bersch, M. J. Stampfer, J.A. Baron, K.M. Egan, W.C. Willett, Physical activity and survival after diagnosis of invasive breast cancer, Cancer Epidemiol. Biomarkers Prev. 17 (2008) 379-386.

[26] M.L. Irwin, A.W. Smith, A. McTiernan, R. Ballard-Barbash, K. Cronin, F.D. Gilliland, R.N. Baumgartner, K.B. Baumgartner, L. Bernstein, Influence of pre- and postdiagnosis physical activity on mortality in breast cancer survivors: the health, eating, activity, and lifestyle study, J. Clin. Oncol. 26 (2008) 3958-3964.

[27] B. Tehard, C.M. Friedenreich, J.M. Oppert, F. Clavel-Chapelon, Effect of physical activity on women at increased risk of breast cancer: results from the E3 $\mathrm{N}$ cohort study, Cancer Epidemiol. Biomarkers Prev. 15 (2006) 57-64.

[28] L. Eklund, M. Bry, K. Alitalo, Mouse models for studying angiogenesis and lymphangiogenesis in cancer, Mol. Oncol. 7 (2013) 259-282.

[29] D.D. Soerensen, S. Clausen, J.B. Mercer, L.J. Pedersen, Determining the emissivity of pig skin for accurate infrared thermography, Comp. Electron. Agric. 109 (2014) 52-58.

[30] F. Denis, P. Bougnoux, W. de Poncheville, M. Prat, R. Catroux, F.O. Tranquart, In vivo quantitation of tumour vascularisation assessed by Doppler sonography in rat mammary tumours, Ultrasound Med. Biol. 28 (2002) 431-437.

[31] D. Forbes, H. Blom, N. Kostomitsopulos, G. Moore, G. Perretta, Euroguide: On the Accommodation and Care of Animals Used for Experimental and Other Scientific Purposes, Federation of European Laboratory Animal Science Associations, London, 2007.

[32] A.I. Faustino-Rocha, A. Silva, J. Gabriel, C.I. Teixeira-Guedes, C. Lopes, R.G. da Costa, A. Gama, R. Ferreira, P.A. Oliveira, M. Ginja, Ultrasonographic, thermographic and histologic evaluation of MNU-induced mammary tumors in female Sprague-Dawley rats, Biomed. Pharmacother. 67 (2013) 771-776.

[33] J. Russo, I.H. Russo, Atlas and histologic classification of tumors of the rat mammary gland, J. Mammary Gland Biol. Neoplasia 5 (2000) 187-200.

${ }^{[34]}$ C.M.L. Chan, B.B.Y. Ma, E.P. Hui, S.C.C. Wong, F.K.F. Mo, S.F. Leung, M.K.M. 
Kam,

A.T.C. Chan, Cyclooxygenase-2 expression in advanced nasopharyngeal carcinoma - a prognostic evaluation and correlation with hypoxia inducible factor 1 alpha and vascular endothelial growth factor, Oral Oncol. 43 (2007) 373-378.

[35] M. Mendonça, E. Soares, L. Stávale, C. Rapôso, A. Coope, E. Kalapothakis, M. Cruz-Höfling, Expression of VEGF and Flk-1 and Flt-1 receptors during blood- brain barrier (BBB) impairment following Phoneutria nigriventer spider venom exposure, Toxins 5 (2013) 2572-2588.

[36] D.J. McCullough, L.M.D. Nguyen, D.W. Siemann, B.J. Behnke, Effects of exercise training on tumor hypoxia and vascular function in the rodent preclinical orthotopic prostate cancer model, J. Appl. Physiol. 115 (2013) 1846-1854.

[37] D. Vordermark, J.M. Brown, Endogenous markers of tumor hypoxiapredictors of clinical radiation resistance? Strahlenther. Onkol. 179 (2003) 801-811.

${ }_{[38]}$ D. McCormick, C. Adamowski, A. Fiks, R. Moon, Lifetime dose-response relationships for mammary-tumor induction by a single administration of $N$-methyl$N$-nitrosourea, Cancer Res. 41 (1981) 1690-1694.

[39] K.S. Whittal-Strange, S. Chadau, W.S. Parkhouse, Exercise during puberty and NMU induced mammary tumorigenesis in rats, Breast Cancer Res. Treat. 47 (1998) $1-8$.

[40] B. teicher, Tumor models for efficacy determination, Mol. Cancer Ther. 5 (2006) 2435-2443.

[41] K.C. Westerlind, H.L. McCarty, P.C. Schultheiss, R. Story, A.H. Reed, M.L. Baier, R. Strange, Moderate exercise training slows mammary tumour growth in adolescent rats, Eur. J. Cancer Prev. 12 (2003) 281-287.

[42] L.W. Jones, N.D. Eves, K.S. Courneya, B.K. Chiu, V.E. Baracos, J. Hanson, L. Johnson, J.R. Mackey, Effects of exercise training on antitumor efficacy of doxorubicin in MDA-MB-231 breast cancer xenografts, Clin. Cancer Res. 11 (2005) 6695-6698.

[43] A.I. Faustino-Rocha, A. Gama, P.A. Oliveira, A. Alvarado, M.J. Neuparth, R. Ferreira, M. Ginja, Effects of lifelong exercise training on mammary (2016), doi:http://dx.doi.org/10.1007/s10238-016-0419-0.

[44] D.A. Kennedy, T. Lee, D. Seely, A comparative review of thermography as a breast cancer screening technique, Integr. Cancer Ther. 8 (2009) 9-16.

[45] B. Brkljacic, D. Miletic, F. Sardanelli, Thermography is not a feasible method for breast bancer screening, Coll. Antropol. 2 (2013) 589-593.

[46] M. Poljak-Blazi, D. Kolaric, M. Jaganjac, K. Zarkovic, K. Skala, N. Zarkovic, Specific thermographic changes during Walker 256 carcinoma development: differential infrared imaging of tumour, inflammation and haematoma, Cancer Detect. Prev. 32 (2009) 431-436.

[47] C. Song, V. Appleyard, K. Murray, T. Frank, W. Sibbett, A. Cuschieri, A. Thompson, Thermographic assessment of tumor growth in mouse xenografts, Int. J. Cancer 121 (2007) 1055-1058.

${ }_{[48]}$ J.F. Head, F. Wang, C.A. Lipari, R.L. Elliott, The important role of infrared imaging in breast cancer-new technology improves applications in risk assessment, detection, diagnosis, and prognosis, IEEE Eng. Med. Biol. Mag. 19 (2000) 52-57. [49] M. Anbar, Clinical thermal imaging today, IEEE Eng. Med. Biol. Mag. 17 (1998) 
$25-33$.

[50] Y.-K. Eddie, S.-C. Fork, A framework for early discovery of breast tumor using thermography with artificial neural network, Breast J. 9 (2003) 341-343.

[51] W. Xie, P. McCahon, K. Jakobsen, C. Parish, Evaluation of the ability of digital infrared imaging to detect vascular changes in experimental animal tumours, Int. J. Cancer 108 (2004) 790-794.

${ }_{[52]}$ D. Hanahan, J. Folkman, Patterns and emerging mechanism of the angiogenic switch during tumorigenesis, Cell 86 (1996) 353-364.

[53] S.B. Fox, K.C. Gatter, A.L. Harris, Tumour angiogenesis, J. Pathol. 179 (1996) 232-237.

[54] J. Folkman, What is the evidence that tumors are angiogenesis dependent, J. Nat. Cancer Inst. 82 (1990) 4-6.

[55] B.P. Schneider, K.D. Miller, Angiogenesis of breast cancer, J. Clin. Oncol. 23 (2005) 1782-1790.

[56] R.S. Herbst, A. Onn, A. Sandler, Angiogenesis and lung cancer: prognostic and therapeutic implications, J. Clin. Oncol. 23 (2005) 3243-3256.

[57] P. Ferroni, A. Spila, F. Martini, R. D'Alessandro, S. Mariotti, G. Del Monte, P. Graziano, O. Buonomo, F. Guadagni, M. Roselli, Prognostic value of vascular endothelial growth factor tumor tissue content of colorectal cancer, Oncology 69 (2005) 145-153.

[58] J. Cui, B.W. Dong, P. Liang, X.L. Yu, D.J. Yu, Effect of c-myc, Ki-67, MMP-2 and VEGF expression on prognosis of hepatocellular carcinoma patients undergoing tumor resection, World J. Gastroenterol. 10 (2004) 1533-1536.

[59] R. Bartoletti, T. Cai, G. Nesi, I. Sardi, M. Rizzo, Qualitative and quantitative analysis of angiogenetic factors in transitional cell bladder carcinoma: relationship with clinical course at 10 years follow-up, Oncol. Rep. 14 (2005) 251-255.

${ }_{[60]}$ T. Meng-Shu, K. Min-Liang, C. Cheng-Chi, W. Ying-Tai, The effects of exercise training on levels of vascular endothelial growth factor in tumor-bearing mice, CCC (2015).

[61] A. Isanejad, A. Alizadeh, S. Shalamzari, H. Khodayari, S. Khodayari, V. Khori, N. Khojastehnjad, MicroRNA-206, let-7a and microRNA-21 pathways involved in the anti-angiogenesis effects of the interval exercise training and hormone therapy in breast cancer, Life Sci. 151 (2016) 30-40.

[62] B. Macneil, L. Hoffmangoetz, Effect of exercise on natural cytotoxicity and pulmonary tumor-metastases in mice, Med. Sci. Sports Exercise 25 (1993) 922928.

[63] B. Macneil, L. Hoffmangoetz, Exercise training and tumor-metastasis in miceinfluence of time of exercise onset, Anticancer Res. 13 (1993) 2085-2088.

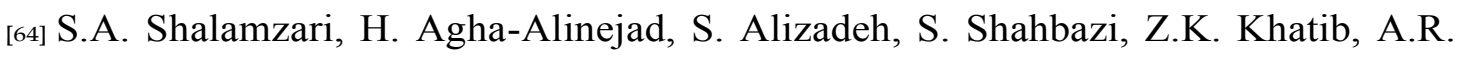
Kazemi, M.A. Saei, N. Minayi, The effect of exercise training on the level of tissue IL6 and vascular endothelial growth factor in breast cancer bearing mice, Iranian J. Basic Med. Sci. 17 (2014) 231-236.

[65] M.R. Zielinski, M. Muenchow, M.A. Wallig, P.L. Horn, J.A. Woods, Exercise delays allogeneic tumor growth and reduces intratumoral inflammation and vascularization, J. Appl. Physiol. 96 (2004) 2249-2256.

[66] B. Restucci, S. Papparella, P. Maiolino, G. De Vico, Expression of vascular 
endothelial growth factor in canine mammary tumors, Vet. Pathol. 39 (2002) 488493.
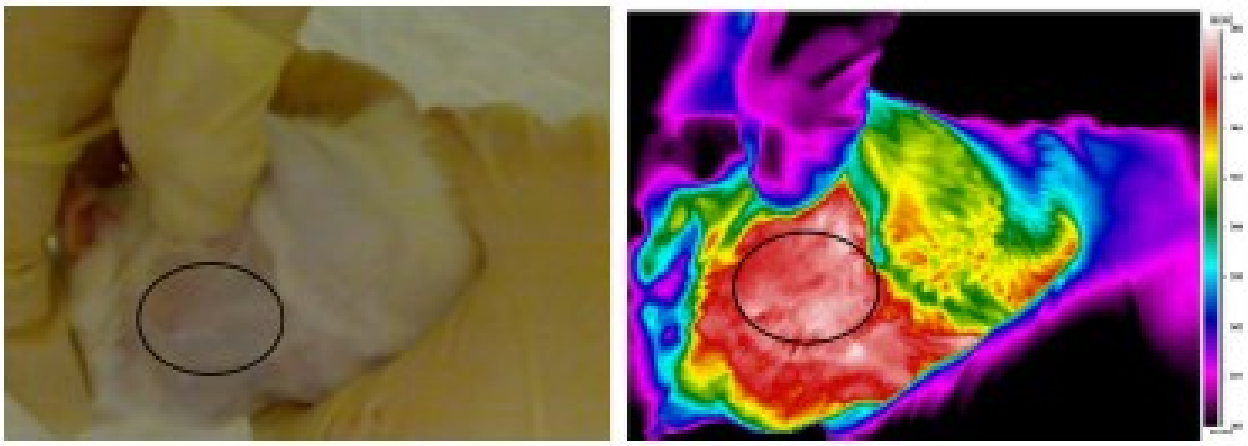

Fig. 1. Visible image (left) and thermographic analysis (right) of a MNU-induced mammary tumor (temperature range $30-38^{\circ} \mathrm{C}$ ). Mammary tumor is delimited in both images by the black circle.

(A)

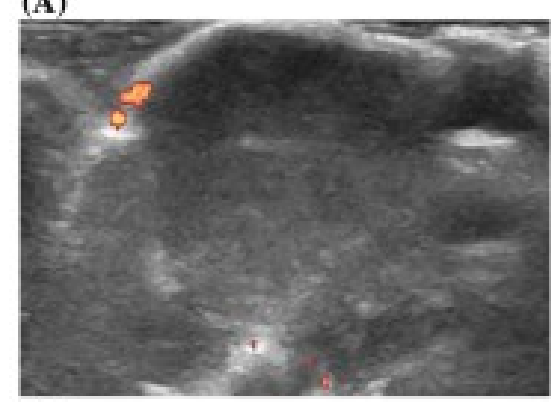

(C)

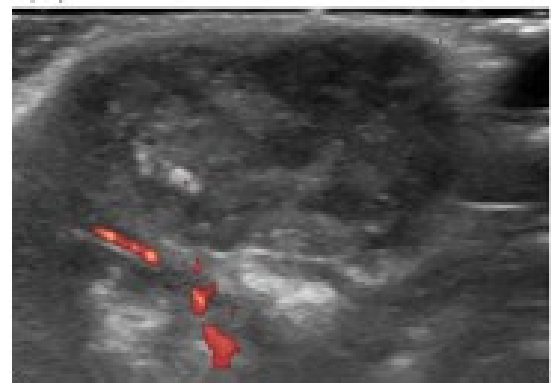

(B)

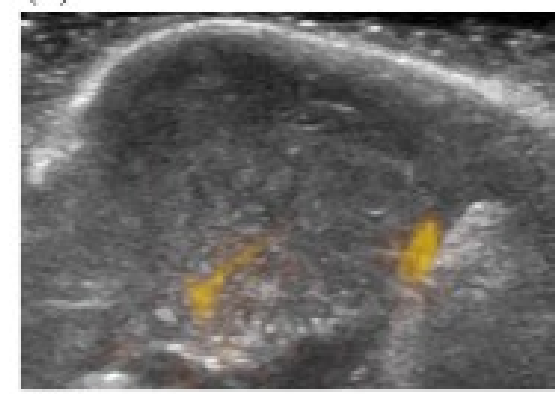

(D)

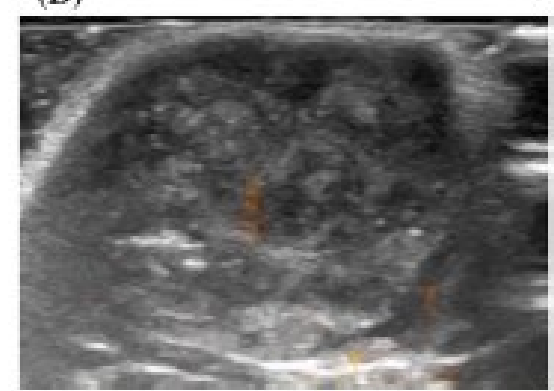

Fig. 2. Evaluation of mammary tumors from MNU sedentary (A and $B$ ) and $M N U$ exercised (C and D) groups by Power Doppler (A and C) and B Flow (B and D). 
(A)

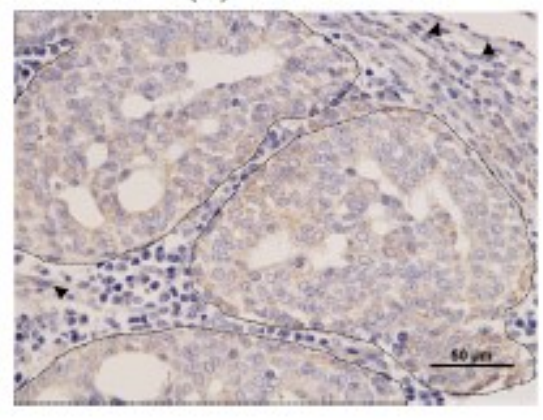

(B)

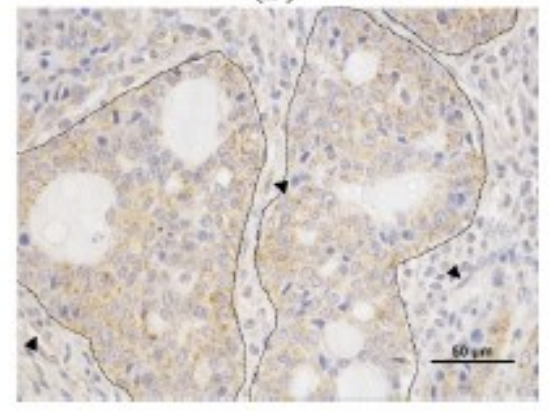

Fig. 3. Immunoexpression of VEGF-A in mammary tumors from MNU sedentary (A) and MNU exercised animals (B). (A) 50.35\% of immunopositive cells for VEGFA (grade 3), moderate staining (++). (B) $64.30 \%$ of immunopositive cells for VEGFA (grade 3), intense staining $(+++)$. Some endothelial cells were immunopositive for VEGF-A (arrowheads). 
Table 1 - Histological classification, weight, volume and area of necrosis of mammary tumors identified in both MNU sedentary and exercised groups taking in account the predominant pattern.

\begin{tabular}{|c|c|c|c|}
\hline \multirow[b]{2}{*}{ Histological classification } & & \multicolumn{2}{|c|}{ Number of tumors } \\
\hline & & MNU sedentary & MNU exercised \\
\hline \multirow{6}{*}{$\begin{array}{l}\text { Non-neoplastic } \\
\text { Benign }\end{array}$} & Epidermal cysts & 1 & 0 \\
\hline & Intraductal papilloma & 0 & 1 \\
\hline & Papillary cystadenoma & 0 & 2 \\
\hline & Tubular adenoma & 1 & 2 \\
\hline & Lactating adenoma & 0 & 1 \\
\hline & Fibroadenoma & 0 & 1 \\
\hline \multirow{11}{*}{$\begin{array}{l}\text { Total: } \\
\text { Malignant }\end{array}$} & & $1^{2}$ & 7 \\
\hline & Papillary noninvasive carcinoma & 13 & 7 \\
\hline & Cribriform noninvasive carcinoma & 5 & 4 \\
\hline & Papillary invasive carcinoma & 4 & 4 \\
\hline & Cribriform invasive carcinoma & 2 & 1 \\
\hline & Comedo invasive cardinoma & 2 & 0 \\
\hline & Total: & $26^{\mathrm{b}}$ & 16 \\
\hline & Total & $28^{c}$ & 23 \\
\hline & Tumors' weight ( $\mathrm{g}$ ) & $5.15 \pm 2.04$ & $8.31 \pm 2.82$ \\
\hline & Tumors' volume $\left(\mathrm{cm}^{3}\right)$ & $4.88 \pm 1.93$ & $7.87 \pm 2.67$ \\
\hline & Area of necrosis $\left(\mathrm{mm}^{2}\right)$ & $0.34 \pm 0.05$ & $0.61 \pm 0.17$ \\
\hline
\end{tabular}

\footnotetext{
$p=0.034$ from MNU exercised group.

$p=0.123$ from $\mathrm{MNU}$ exercised group.
}

$p=0.484$ from MNU exercised group. 
Table 2 - Thermographic, ultrasonographic and immunohistochemical evaluation of mammary tumors in both groups MNU sedentary and exercised (mean \pm S.E.).

\begin{tabular}{|c|c|c|c|}
\hline \multirow[b]{2}{*}{ Parameter } & & \multicolumn{2}{|l|}{ Groups } \\
\hline & & MNU sedentary & MNU exercised \\
\hline \multirow{4}{*}{ Temperature $\left({ }^{\circ} \mathrm{C}\right)$} & Maximum & $37.46 \pm 0.21^{2}$ & $37.43 \pm 0.38^{2}$ \\
\hline & Minimum & $34.12 \pm 0.42$ & $32.78 \pm 1.20$ \\
\hline & Thermal amplitude & $3.35 \pm 0.54$ & $4.66 \pm 1.02$ \\
\hline & Mean & $36.28 \pm 0.16$ & $35.96 \pm 0.55$ \\
\hline \multirow[t]{2}{*}{ CPD $(\%)$} & Power Doppler & $1.30 \pm 0.29$ & $1.47 \pm 0.27^{\mathrm{b}}$ \\
\hline & B Flow & $2.40 \pm 0.52$ & $3.68 \pm 0.88$ \\
\hline VEGF-A immunoexpression by GIMP ( $(\%)$ & & $59.91 \pm 3.11$ & $66.04 \pm 4.65$ \\
\hline MVD (microvessel density) & & $11.82 \pm 1.09^{c}$ & $18.35 \pm 2.93$ \\
\hline
\end{tabular}

CPD; color pixels density.

2 Statistically different from minimum temperature $(p<0.05)$.

b Statistically different from B Flow $(p<0.05)$.

${ }^{c}$ Statistically different from MNU exercised group $(p<0.05)$. 
Table 3 - Semi-quantitative evaluation of VEGF-A immunoexpression in neoplastic cells of MNU-induced mammary tumors from sedentary and exercised animals (only the data from malignant tumors were compared between groups).

\begin{tabular}{|c|c|c|c|c|c|}
\hline Score/Croup & & & & MNU sedentary $(\mathrm{n}=28)$ & MNU exerdised $(n-23)$ \\
\hline \multirow[t]{7}{*}{ Neoplastic cells } & Immunopositive cells & 0 & $a x$ & $a(a x)$ & $a(c)$ \\
\hline & & 1 & $1-24 x$ & $3(107 x)^{3}$ & $2(8.7 x)$ \\
\hline & & 2 & $25-498$ & $3(10.7 x)^{3}$ & $4(124 x)$ \\
\hline & & 4 & $>75 x$ & $9(321 \%)$ & $11(478 x)^{p}$ \\
\hline & Intensity of immunostaining & 0 & Unstained & $0(a x)$ & $a(a x)$ \\
\hline & & + & Weak & $6(2.4 X)$ & $11(478 x)$ \\
\hline & & + & Moderate & $20(714 x)^{-d}$ & $5(21.7 x)$ \\
\hline
\end{tabular}

A Statistically different from Score $3(p<005)$.

b Statistically different from Score $1(p<005)$

satistically different from weak $(+)$ and intense staining $(+++)(p<005)$

a Statistically different from moderate staining $(+)$ in MNU exerdised group $(p<0,05)$

Table 4 - Correlation between data from thermography, ultrasonography and immunohistochemistry.

\begin{tabular}{|c|c|c|c|c|c|c|c|c|}
\hline Parameter & $\begin{array}{l}\text { Tumors' } \\
\text { volume }\end{array}$ & Maximum $\mathrm{T}^{\circ}$ & Minimum $T^{\circ}$ & Mean $\mathrm{T}^{\circ}$ & $\begin{array}{l}\text { Thermal } \\
\text { amplitude }\end{array}$ & CPD PDI & CPD B Flow & MVD \\
\hline Tumors' volume & - & $\begin{array}{l}0.113 \\
(p=0.626)\end{array}$ & $\begin{array}{l}-0.491^{*} \\
(p=0.024)\end{array}$ & $\begin{array}{l}-0.197 \\
(p=0.393)\end{array}$ & $0.568^{*}(p=0.007)$ & $0.098(p=0.599)$ & $0.007(p=0.969)$ & $-0.160(p=0.281)$ \\
\hline Maximum $\mathrm{T}^{\circ}$ & - & - & $0.362(p-0.107)$ & $\begin{array}{l}0.870^{\circ} \\
(p=0.000)\end{array}$ & $\begin{array}{l}-0.028 \\
(p=0.904)\end{array}$ & $\begin{array}{l}-0.250 \\
(p=0.275)\end{array}$ & $0.001(p=0.996)$ & $\begin{array}{l}-0.023 \\
(\mathrm{pp}=0.922)\end{array}$ \\
\hline Minimum $\mathrm{T}^{\circ}$ & - & - & - & $\begin{array}{l}0.731^{\circ} \\
(p-0.000)\end{array}$ & $\begin{array}{l}-0.942^{*} \\
(p=0.000)\end{array}$ & $0.251(p-0.273)$ & $0.137(p=0.553)$ & $-0.184(p-0.425)$ \\
\hline Mean $\mathrm{T}^{\circ}$ & - & - & - & - & $\begin{array}{l}-0.472^{*} \\
(p=0.031)\end{array}$ & $0.028(p=0.905)$ & $0.108(p-0.641)$ & $-0.195(p=0.398)$ \\
\hline $\begin{array}{l}\text { Thermal } \\
\text { amplitude }\end{array}$ & - & - & - & - & - & $\begin{array}{l}-0.270 \\
(p=0.237)\end{array}$ & $\begin{array}{l}-0.369 \\
(p=0.099)\end{array}$ & $0.195(p=0.396)$ \\
\hline CPD PDI & - & - & - & - & - & - & $0.012(p=0.945)$ & $0.073(p=0.740)$ \\
\hline CPD B Flow & - & - & - & - & - & - & - & $0.359(p=0.101)$ \\
\hline MVD & - & - & - & - & - & - & - & - \\
\hline
\end{tabular}

- These correlations were considered statistically significant $(p<0.05)$. 